\title{
Does Current Diabetes Technology Improve Metabolic Control? A Cross-Sectional Study on the Use of Insulin Pumps and Continuous Glucose Monitoring Devices in a Nationwide Pediatric Population
}

\author{
Heiko Bratke (D) · Hanna D. Margeirsdottir · Jörg Assmus (D) • \\ Pål R. Njølstad (D) · Torild Skrivarhaug (D)
}

Received: June 21, 2021 / Accepted: July 21, 2021 / Published online: August 13, 2021

(c) The Author(s) 2021

\section{ABSTRACT}

Objective: To examine the use of multiple daily injections (MDI), insulin pumps, self-measured blood glucose (SMBG), and continuous glucose monitoring (CGM) systems, and their association with glycated hemoglobin (HbA1c), diabetic ketoacidosis (DKA), and severe hypoglycemia.

Methods: In a pediatric population-based nationwide cross-sectional study, we analyzed data from 2623 participants up to 18 years of age with type 1 diabetes, using 2017 annual data from the Norwegian Childhood Diabetes Registry. HbA1c was adjusted for age, gender,

Supplementary Information The online version contains supplementary material available at https:// doi.org/10.1007/s13300-021-01127-6.

H. Bratke $(\square)$

Department of Pediatrics, Haugesund Hospital, Fonna Health Trust, Postbox 2170, 5504

Haugesund, Norway

e-mail: heiko.bratke@uib.no

H. Bratke · P. R. Njølstad

Department of Clinical Science, Center for Diabetes Research, University of Bergen, Bergen, Norway

H. Bratke · H. D. Margeirsdottir · T. Skrivarhaug Oslo Diabetes Research Centre, Oslo, Norway

H. D. Margeirsdottir · T. Skrivarhaug Division of Childhood and Adolescent Medicine, Oslo University Hospital, Oslo, Norway and diabetes duration. Using a linear mixed-effects model, we assessed HbA1c and the incidence of DKA and severe hypoglycemia according to the use of MDI, insulin pumps, SMBG, and CGM.

Results: We observed that $74.7 \%$ of participants were using an insulin pump and 52.6\% were using a CGM system. Mean HbA1c was $7.8 \%$ (62 $\mathrm{mmol} / \mathrm{mol})$. The HbA1c of pump users was 0.14 percentage points (pp) higher than that of MDI users. Fewer pump users than MDI users achieved an HbA1c of $<7.5 \%$ (38.3 vs. 41.6\%). CGM users had a 0.18 pp lower HbA1c than SMBG users, with 40.5 and $38.0 \%$, respectively, achieving an HbA1c of $<7.5 \%$. The incidence of severe hypoglycemia or hospitalization due to DKA was not different in pump and CGM users compared with nonusers. Compared with other insulin pumps, patch

J. Assmus

Centre for Clinical Research, Haukeland University

Hospital, Bergen, Norway

P. R. Njølstad

Children and Adolescent Clinic, Haukeland

University Hospital, Bergen, Norway

T. Skrivarhaug

Faculty of Medicine, Institute of Clinical Medicine, University of Oslo, Oslo, Norway

T. Skrivarhaug

Division of Childhood and Adolescent Medicine, The Norwegian Childhood Diabetes Registry, Oslo University Hospital, Oslo, Norway 
pump use was associated with a significantly lower odds ratio for DKA.

Conclusions: Despite the broad use of diabetes technology, as many as $61 \%$ of our pediatric cohort did not reach the HbA1c target recommended by the International Society for Pediatric and Adolescent Diabetes (ISPAD). Lower HbA1c was associated with CGM use but not with insulin pump use. Acute complications were not less frequent in the groups using insulin pumps or CGM compared with those using MDI and SMBG. Further research is required to explore the lower incidence of DKA among patch pump users.

Trial Registration: ClinicalTrials.gov identifier NCT04201171.

Keywords: Diabetes Mellitus; Type 1; Hypoglycemia/prevention and control; Diabetic ketoacidosis; Glycosylated hemoglobin; Continuous glucose monitoring; Continuous subcutaneous insulin infusion

\section{Key Summary Points}

Knowledge on the use of continuous subcutaneous insulin infusion (CSII) and continuous glucose monitoring (CGM) under real-world conditions is limited, and existing studies with different study designs have shown different results on the possible effect of these devices on glycated hemoglobin (HbA1c), diabetic ketoacidosis, and severe hypoglycemia in persons with type 1 diabetes.

The overall use of CSII was not associated with a higher proportion of children and adolescents with $\mathrm{HbA} 1 \mathrm{c}<7.5 \%$ ( $58 \mathrm{mmol} / \mathrm{mol}$ ) or lower mean HbA1c. The use of continuous glucose monitoring, however, was associated with a 0.15 percentage point lower HbA1c. Acute complications were not less common when using CSII or CGM compared with nonuse. Patch pumps might reduce the risk of diabetic ketoacidosis.
Expectations for the use of technical devices, at least those prevalent in 2017, should be modest. Insulin pumps and CGM systems that are more user-friendly and more sophisticated, especially newer closed-loop systems, might lead to better metabolic control than the devices used in our 2017 cohort.

\section{INTRODUCTION}

Major goals in the treatment of type 1 diabetes in children are reducing disease burden and treatment-associated stress and avoiding acute adverse effects and long-term complications. Low glycated hemoglobin (HbA1c) levels and a low incidence of episodes of diabetic ketoacidosis and severe hypoglycemia reflect the achievement of these goals. In October 2018, the International Society for Pediatric and Adolescent Diabetes (ISPAD) recommended a target of HbA1c of $<7.0 \%(53 \mathrm{mmol} / \mathrm{mol})$ [1]. Most children and adolescents with type 1 diabetes do not meet this target, according to data from national and international diabetes registries [1-5].

Recently, the use of continuous subcutaneous insulin infusion (CSII) and continuous glucose monitoring (CGM) has increased sharply, at least in developed countries [6-8]. Results from large international registries have indicated an association between the use of CSII and lower HbA1c, compared with the regimen of multiple daily injections (MDI) $[9,10]$. By contrast, a recent randomized controlled trial on the use of CSII in a pediatric population in the first year of diabetes concluded that CSII was not beneficial compared with MDI [11]. The combination of pump therapy with CGM (sensor-augmented pump) has been shown to be associated with lower HbA1c compared to MDI in combination with self-measured blood glucose (SMBG) [12]. CGM was shown to be associated with lower $\mathrm{HbA1c}$ in both adolescents and young adults in a randomized controlled 
trial [13] and in adults in an observational study [14].

In recent years, rapid technological developments have been made in CSII and CGM systems. Different companies offer systems with different properties, and their use varies worldwide due to local preferences, access to the product, financial aspects, organization of the national healthcare system, and reimbursement. A knowledge gap exists regarding the possible effect of these various systems on acute incidents and long-term complications, since existing studies are based on few and selected participants and a short follow-up time. In addition, technological development often outpaces the speed of publication [15-18].

The Norwegian Health Care System fully reimburses CSII and CGM systems for all children and adolescents with type 1 diabetes. Consequently, the use of CSII in Norwegian children increased sharply after 2000, from 9\% in 2001 to $73 \%$ in 2017 [19]. Many children start with CSII right from diabetes onset. The main indication for insulin pumps and CGM in the pediatric age group in Norway is not to improve glucose control in individuals failing on MDI but to give broad access to the advantages of the devices, such as, for example, fewer injections and finger sticks, which is crucial in the pediatric age group. In Norway, eligible CSII and CGM devices are defined in a national contract handled by the Regional Health Trusts' purchasing department (HINAS). On the basis of a broad and detailed evaluation of the item's qualities and costs, a multidisciplinary expert panel issues national recommendations for the use of different types of CSII and CGM in pediatric care. The device with the best cost-benefit ratio should be used as the first priority. At the time of data collection for this publication (2017), the first-priority systems recommended by HINAS for CSII and CGM were the Accu-Chek Combo (Roche Diagnostics, Indianapolis, IN, USA) and the Freestyle Navigator (Abbott Diabetes Care, Alameda, CA, USA), respectively. As these recommendations are not binding, clinicians and users are free to choose eligible devices according to what they find more suitable for the individual user's needs.
The aims of this study were to examine the use of technical devices in the Norwegian pediatric population and to assess the association of MDI, CSII, and CGM, alone or in combination, with $\mathrm{HbA1c}$, hospitalization caused by diabetic ketoacidosis (DKA), and incidence of severe hypoglycemia.

\section{METHODS}

This population-based nationwide cross-sectional study used data from the Norwegian Childhood Diabetes Registry (NCDR).

\section{Sample}

In Norway, all children with diabetes up to 18 years of age should, according to a recommendation from the Norwegian Directorate of Health, receive their diabetes follow-up in a pediatric department. All pediatric departments report standardized clinical data on their patients to the NCDR at diabetes onset and annually thereafter. As part of the annual registration in NCDR, HbA1c is analyzed at a laboratory with Diabetes Control and Complications Trial (DCCT)-standardized HbA1c measurement program once yearly, one HbA1c measurment per child. The data completeness of NCDR is high, with $99 \%$ data completeness at the individual level at diabetes onset and 98\% on annual examinations [19]. In our study, we included all individuals with type 1 diabetes who participated in the 2017 annual registration. We excluded individuals aged > 18 years at the time of data registration.

\section{Sample Characteristics}

As shown in Table 1, the 2017 cohort comprised 2623 children and adolescents with type 1 diabetes $(45.4 \%$ of whom were female). Mean age was 12.6 (standard deviation [SD] 3.8, range 1.7-18) years, and mean diabetes duration was 5.1 (SD 3.6) years. Insulin pumps in use at the time of the data collection were the Accu-Chek Aviva Combo (Roche Diagnostics), Paradigm pumps (models 522, 554, 715, and 722; 
Table 1 Study sample characteristics

\begin{tabular}{|c|c|c|c|c|}
\hline Study characteristics & Valid $N(\%)$ & Age (years), mean (SD) & Female, $N(\%)$ & $\begin{array}{l}\text { Diabetes duration (years), } \\
\text { mean (SD) }\end{array}$ \\
\hline Total pediatric cohort & 2623 & $12.6(3.8)$ & $1192(45.4 \%)$ & $5.1(3.6)$ \\
\hline \multicolumn{5}{|l|}{ Devices } \\
\hline CSII & $1918(74.7 \%)$ & $12.4(3.8)$ & $898(46.8 \%)$ & $5.2(3.5)$ \\
\hline MDI & $651(25.3 \%)$ & $13.2(3.6)$ & $267(41 \%)$ & $4.7(3.6)$ \\
\hline CGM & $1321(52.6 \%)$ & $11.5(3.9)$ & $615(46.6 \%)$ & $4.7(3.4)$ \\
\hline No CGM & $1190(47.4 \%)$ & $13.7(3.3)$ & $517(43.4 \%)$ & $5.4(3.7)$ \\
\hline CSII + CGM & $1124(58.6 \%)$ & $11.4(3.9)$ & $537(47.8 \%)$ & $4.8(3.3)$ \\
\hline $\mathrm{MDI}+\mathrm{CGM}$ & $196(31.6 \%)$ & $12(3.8)$ & $78(39.8 \%)$ & $4.6(3.7)$ \\
\hline CSII-CGM & $765(40.5 \%)$ & $13.6(3.3)$ & $346(45.2 \%)$ & $5.9(3.7)$ \\
\hline MDI-CGM & $424(68.4 \%)$ & $13.8(3.3)$ & $171(40.3 \%)$ & $4.6(3.6)$ \\
\hline \multicolumn{5}{|l|}{ CSII device types ${ }^{a}$} \\
\hline Accu-Check Aviva Combo & $98(5.2 \%)$ & $12.7(3.9)$ & $38(38.8 \%)$ & $4.1(3.1)$ \\
\hline Medtronic Paradigm & $774(40.8 \%)$ & $12.9(3.7)$ & $342(44.2 \%)$ & $6.3(3.8)$ \\
\hline Animas Vibe & $396(20.9 \%)$ & $11.7(4)$ & $176(44.4 \%)$ & $3.9(3)$ \\
\hline Medtronic $640 \mathrm{G}$ & $119(6.3 \%)$ & $11.1(4.1)$ & $58(48.7 \%)$ & $4.9(3.7)$ \\
\hline Omnipod & $510(26.9 \%)$ & $12.4(3.5)$ & $272(53.3 \%)$ & $5.1(3.1)$ \\
\hline \multicolumn{5}{|l|}{ CGM device types ${ }^{\mathrm{a}}$} \\
\hline Freestyle Navigator & $55(4.6 \%)$ & $12.1(3.5)$ & $31(56.4 \%)$ & $4.7(3.5)$ \\
\hline Dexcom G4 & $587(49.5 \%)$ & $11.3(3.9)$ & $267(45.5 \%)$ & $4.4(3.1)$ \\
\hline Medtronic Enlite & $387(32.6 \%)$ & $11.6(4)$ & $174(45 \%)$ & $5.2(3.7)$ \\
\hline Freestyle Libre & $157(13.2 \%)$ & $11.9(3.7)$ & $66(42 \%)$ & $4.7(3.4)$ \\
\hline
\end{tabular}

CSII Continuous subcutaneous insulin infusion, CGM continuous glucose monitoring, $M D I$ multiple daily injections, $S D$ standard deviation

a See text for manufacturers of devices

Medtronic plc, Dublin, Ireland), MiniMed 640G pump system (Medtronic), the Animas Vibe (Rubin Medical AB, Limhamn, Sweden), and the Omnipod (Insulet Corp., Acton, MA, USA). CGM systems in use were the Freestyle Navigator and Freestyle Libre (Abbott Diabetes Care), Dexcom G4 (Dexcom Inc., San Diego, CA, USA), and Enlite (Medtronic). MDI treatment was solely given with an insulin pen.

\section{Measures}

We evaluated the patients' present diabetes management according to their use of technical devices, such as CSII and CGM, as a reflection of insulin delivery, glucose control, and a combination thereof.

The primary outcome measures were $\mathrm{HbA1c}$, incidence of DKA, and severe hypoglycemia, as defined by ISPAD [20-22]. The predictors were 
the use of MDI, CSII, different CSII models, SMBG, CGM, and different CGM models, as mentioned above.

Because HbA1c analysis was centralized at a DCCT-standardized laboratory (Aker Laboratory, Department of Medical Biochemistry, Oslo University Hospital, Oslo, Norway), HbA1c values from all participants were comparable. The laboratory has used an ion-exchange HPLC method from Tosoh Bioscience (San Francisco, CA, USA) since 2007; specifically, the Tosoh G7 and G8 systems were used up to after June 12, 2017, respectively (coefficient of variation $<2.5 \%$ ). The HbA1c values were adjusted for age, gender and diabetes duration. We fitted linear and quadratic models with and without interactions; the linear model without interactions was found to have better fit and was used. Adjusted HbA1c values were used for the analyses.

The study sample was categorized regarding the method of insulin delivery (CSII or MDI), glucose control (CGM yes/no), and combinations thereof. In the CSII and CGM groups, participants were categorized into subgroups depending on the exact type of device they used (i.e., manufacturer and device model).

\section{Statistical Analysis}

We assessed the association between $\mathrm{HbA1c}$ and the use of CSII (vs. MDI) and CGM (vs. SMBG) by using the linear mixed-effects (LME) model with $\mathrm{HbA1c}$ as the dependent variable and CSII and CGM as predictors. We estimated the univariate models for each predictor, the model including both predictors, and the model with their interaction. The hospital delivering follow-up was used as a random intercept in all models. For the analysis of the different CSII and CGM types, we used the same approach but compared the use of each device against using the first priority choice. For evaluating the incidence of acute complications, we used logistic regression with the technical devices as predictors. Generally, very little data were missing; hence, the data were handled by listwise deletion. The general significance level was set to 0.05 . All computations were performed in
$\mathrm{R}$ 4.0.2 [23] using the packages nlme [24] and emmeans [25]. The graphics were derived in $\mathrm{R}$ 4.0.2 and MATLAB 2019a (MathWorks, Natick, MA, USA).

\section{Compliance with Ethics Guidelines}

To be registered in NCDR, all patients older than 11 years, and their parents have to sign an informed consent document. When the patients are 16 years, they have to re-sign the same informed consent document. Access to the data for this study was given to two of the authors (Heiko Bratke and Jörg Assmus), in accordance to the internal standards of the data owner (NCDR). The study was approved by the Regional Committee for Medical and Health Research Ethics (reference no. 2016/1613/REC West, dated 11 November 2016), the data delivery committee of the Norwegian Childhood Diabetes Registry (NCDR, dated 8 May, 2017), and the Personal Data Security Office (Personvernombudet [PVO]) at Oslo University Hospital (dated 28 February 2017). The study was performed in accordance with the Helsinki Declaration of 1964 and its later amendments. The study was registered at clinicaltrials.gov (ref. no. NCT04201171).

\section{RESULTS}

\section{Use of CSII and CGM}

Of the 2623 participants, 54 (2.1\%) did not have data on the type of insulin delivery device used. Of the remaining 2569 participants, 1918 (74.7\%) used CSII device and 651 (25.3\%) used MDI. The exact type of CSII device was reported in 1897 patients (data missing for 1.1\% [21/ 1918]), as follows: Accu-Chek Aviva Combo (5.2\%), Medtronic Paradigm models (40.8\%), Medtronic MiniMed 640G (6.3\%), Animas Vibe (20.9\%), and Omnipod patch pump (26.9\%). Regarding the use of CGM, data were missing in $112(4.3 \%)$ of 2623 participants. Of the remaining 2511 participants, 1321 used CGM (52.6\%). The exact type of CGM was registered for 1186 participants (data missing for $10.2 \%$ 
[135/1321]), and the CGM systems in use during the study period were from Abbott (Freestyle Navigator [4.6\%], Freestyle Libre [13.2\%]), Medtronic (Enlite [32.6\%]), and Dexcom (G4 [49.5\%]). More detailed information is provided in Electronic Supplementary Material (ESM) Table 1.

\section{Glycated Hemoglobin}

Glycated hemoglobin was centrally analyzed in 2555 of 2623 patients, and data were missing in 68 patients $(2.6 \%)$. The mean HbA1c of the study cohort was $7.83 \%(62 \mathrm{mmol} / \mathrm{mol})$. Of these 2555 patients, only $39.5 \%$ reached the former ISPAD HbA1c target of $<7.50 \%$ $(58 \mathrm{mmol} / \mathrm{mol})$ and $20 \%$ reached the revised 2018 target [1] of $<7.00 \%(53 \mathrm{mmol} / \mathrm{mol})$.

Figure 1 presents detailed information on adjusted mean HbA1c and percentage of HbA1c under the ISPAD treatment goals from 2014 and 2018, respectively, in the different treatment groups. Electronic Supplementary Material (ESM) Table 1 presents the HbA1c raw data and data adjusted for age, gender, and diabetes duration. Figure 2 illustrates the results from the LME model analysis, based on adjusted data, in an estimated marginal means plot.

Insulin pump users had higher HbA1c than MDI users $(B=+0.14 \mathrm{pp} ; \quad 95 \%$ confidence interval $[\mathrm{CI}] 0.04,0.24 ; p=0.008$ ) (Fig. 2). Fewer pump users than MDI users achieved an HbA1c of $<7.5 \%$ ( 38.3 vs. $41.6 \%$ ). LME model analysis revealed a lower mean HbA1c in participants using the Medtronic Paradigm $(B=-0.31 \mathrm{pp}$; $95 \%$ CI $-0.53,-0.09 ; p=0.006)$ and Omnipod pumps $(B=-0.33$ pp; $95 \%$ CI -0.56 , $-0.10 ; p=0.005$ ) than in those using the firstpriority pump by Roche. Users of the Medtronic 640G and Animas pumps had mean HbA1c comparable with Roche pump users.

LME model analysis of the adjusted data revealed that CGM users had lower HbA1c $(B=-0.18 \mathrm{pp} ; \quad 95 \% \quad \mathrm{CI} \quad-0.27, \quad-0.09 ;$ $p<0.001$ ) (Fig. 2). More CGM users than nonusers achieved an HbA1c of $<7.5 \%$ (40.5 vs. $38.0 \%)$. A significant difference in HbA1c was noted between the users of the Freestyle Navigator and the users of the Dexcom G4 users
Fig. 1 Outcome measures included HbAlc (adjusted for age, gender, and diabetes duration), incidence of diabetic ketoacidosis, and hospitalization for severe hypoglycemia in the different user groups of the insulin delivery (CSII, $\mathrm{MDI}$ ) and glucose monitoring (CGM yes/no) systems (a) and according to the specific CSII and CGM models (b). HbA1c groups, incidence of diabetic ketoacidosis and severe hypoglycemia: Bars represent the number of individuals, and the percentage of the total cohort is given on the $x$-axis. CSII Continuous subcutaneous insulin infusion, $C G M$ continuous glucose monitoring, $\mathrm{HbAlc}$ glycated hemoglobin, $M D I$ multiple daily injections

$(B=0.30 \mathrm{pp} ; 95 \%$ CI $0.04,0.56 ; p=0.026)$, as well as between the Freestyle Navigator users and the Medtronic Enlite users $(B=0.27 \mathrm{pp}$; 95\% CI 0.01, 0.53; $p=0.046$ ) (Fig. 2). The association between mean HbA1c and CSII or CGM at the hospital level is illustrated in ESM Fig. 1.

\section{Incidence of DKA Leading to Hospitalization}

We identified 67 incidents of DKA per year, reflecting $2.6 \%$ of the study sample $(n=2544$, data missing in 79 patients). Logistic regression analysis indicated no difference in the incidence of DKA related to the general use of CSII or CGM. However, a significantly lower odds ratio (OR) for DKA was detected in the Omnipod user group (OR 0.18; 95\% CI 0.06, 0.54; $p=0.002)$. No other pump or CGM type was associated with a different incidence of DKA.

\section{Incidence of Severe Hypoglycemia}

The incidence of reported severe hypoglycemia was 3.5\% (90/2541 patients; data missing in 82 patients). Logistic regression analysis adjusted for confounders did not indicate any associations between severe hypoglycemia and use of CSII, CGM, or any special type of devices.

\section{DISCUSSION}

In this nationwide population-based pediatric cohort with liberal and free access to different 
a
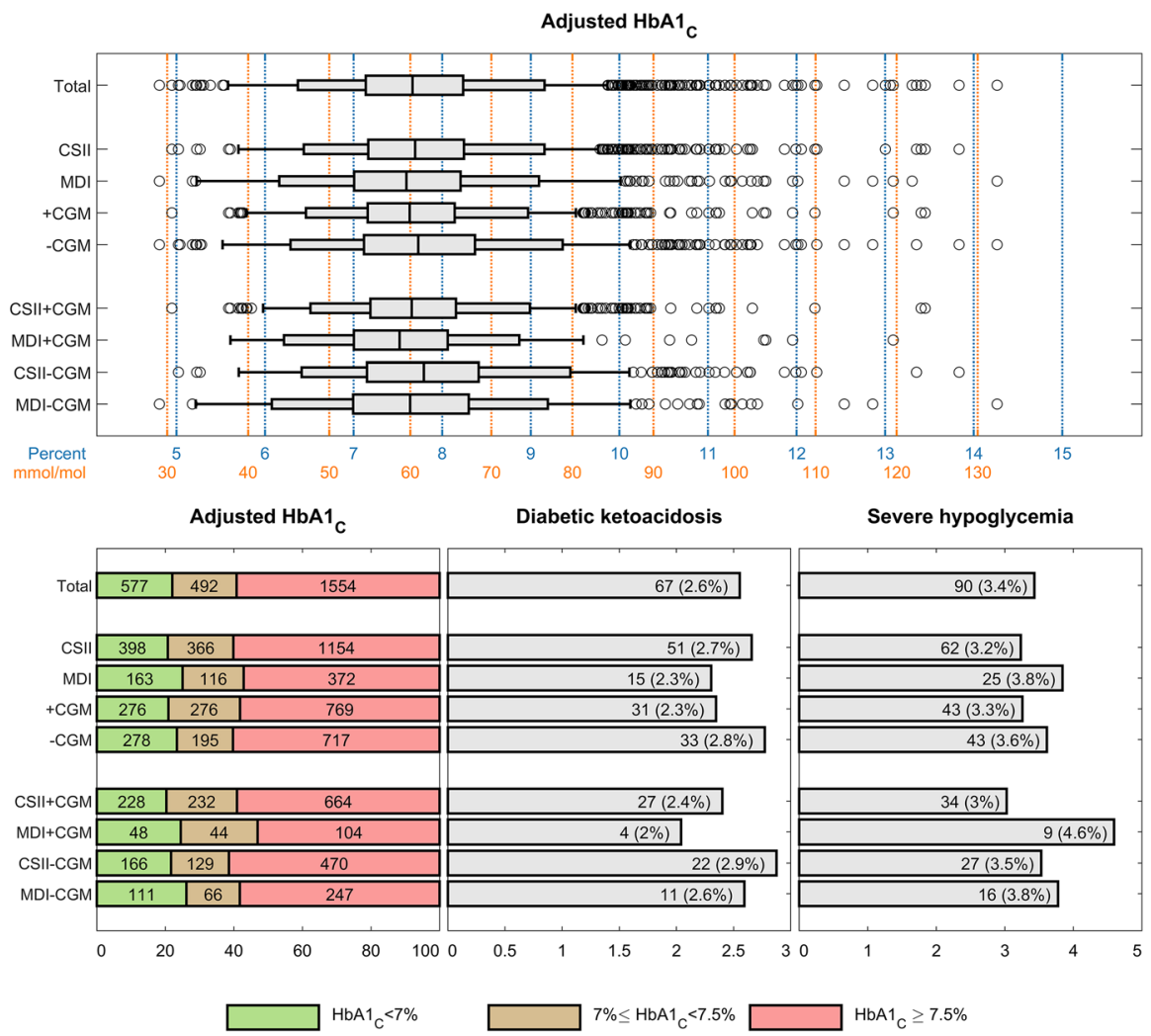

b

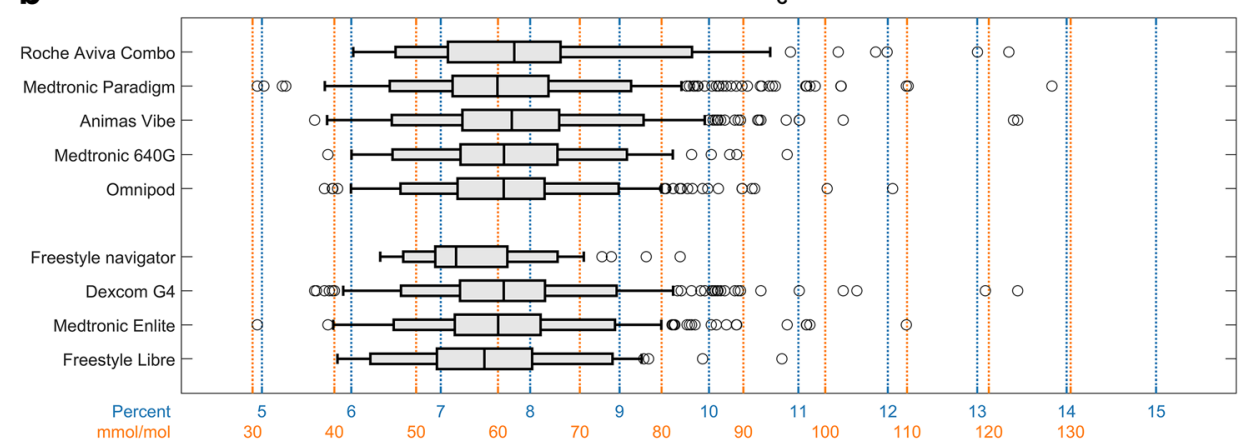

Adjusted HbA1 $c$

Diabetic ketoacidosis

Severe hypoglycemia

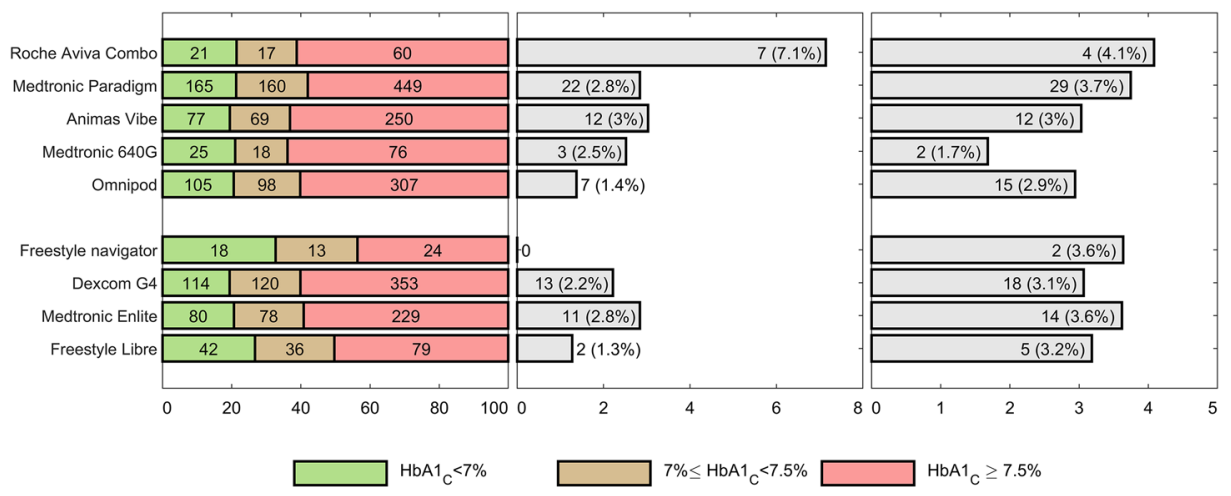




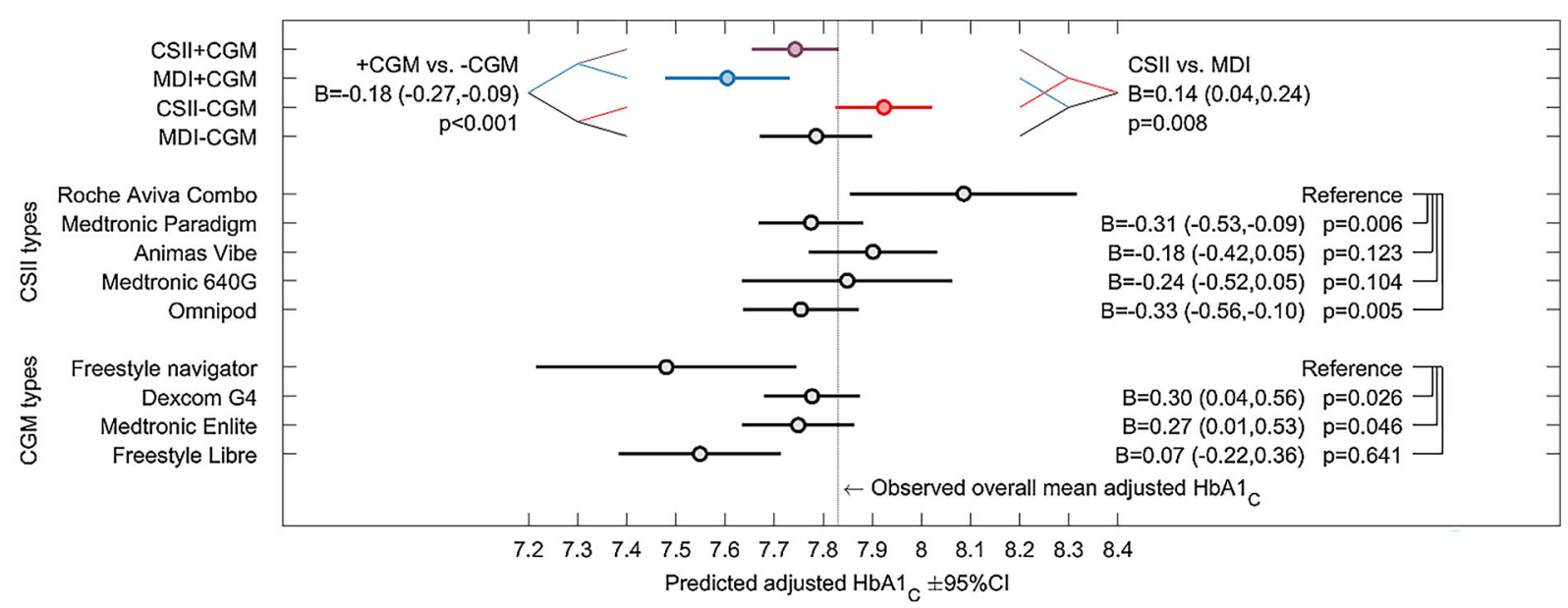

Fig. 2 Estimated marginal means plot with predicted adjusted HbAlc for CSII and CGM and the different models in use. CI Confidence interval

CSII and CGM devices, the overall use of insulin pumps was associated with higher HbA1c. The general use of CGM was, however, associated with a slightly lower HbA1c. There was no correlation between the incidence of acute complications and the use of CSII and CGM in general. Patch pump users were at significantly lower risk for DKA than the users of any other pump.

The possible impact of technical devices, such as CSII and CGM devices, depends mainly on three factors: users (i.e., persons with type 1 diabetes), healthcare professionals delivering diabetes care and education, and the device itself. In a real-world setting, the user's motivation for good metabolic control, general diabetes knowledge, diabetes education, and more specific education in using and understanding the device's functions can differ widely between users at one hospital site as well as between hospitals. Similarly, considerable variations can be seen in healthcare professionals' motivation, knowledge, skills, and possibility to deliver excellent care and education in the hospital setting. The diabetes teams have to not only master different devices but also be able to teach and train the patient and eventually the patient's caretaker(s) in how to use them. A prerequisite to accessing the full potential of these devices is sound knowledge of the healthcare worker in handling these challenges in a structured way; consequently, the healthcare system must have a plan to provide this knowledge to and to train staff providing diabetes care. Thus, the characteristics of the devices should therefore be considered as only partly responsible for any outcome measure.

\section{CSII}

In Norway, insulin pumps are given freely to children and adolescents to reduce the amount of needle sticks and to deliver a more physiological basal dose-and not just to achieve better glycemic control in cases where MDI fails. Individuals in our cohort did not have to achieve any defined results to start or stay on CSII. Consequently, only approximately onefourth of the patients were injecting insulin by pen.

Our results on pump usage are not in line with results reported in earlier cross-sectional or longitudinal studies. Sherr et al. [10] examined the HbA1c results in a similar cross-sectional cohort of three large pediatric registries, including data from Germany/Austria, England/ Wales, and the USA. In the time frame of 2010-2012, they revealed a $0.5 \mathrm{pp}$ $(5.5 \mathrm{mmol} / \mathrm{mol})$ lower mean HbA1c in participants using CSII. Burckhardt et al. [26] followed a large pediatric cohort of pump users and matched pairs using MDI over a 10-year period; as such, it is the largest and longest longitudinal 
case-control study reported to date, with 463 matched pairs. Children using CSII had a $0.4 \mathrm{pp}$ ( $4 \mathrm{mmol} / \mathrm{mol}$ ) lower HbA1c during the first 6 years of follow-up. However, significant differences in HbA1c were not observed in later years, primarily because of the increasingly lower number of patients under follow-up [26]. Analysis of cross-sectional data from the international pediatric diabetes registry SWEET collected in 2017 revealed that lower HbA1c and lower DKA incidence were associated with the use of insulin pumps [27]. By contrast, our data show only a slightly higher HbA1c in the pump user group. In 2019, Blair et al. [11] published the first randomized controlled trial comparing CSII and MDI in a pediatric population. The number of participants in the CSII arm was rather low $(n=144)$, the time of individual follow-up short (12 months after diagnosis), and the recruiting period quite long (2011-2017). The study had a high percentage of participants who declined to participate, and a high dropout rate of participants who, after randomization, were not getting their preferred treatment. The results did indicate a lack of impact of CSII on HbA1c after the first year. In addition to Blair et al.'s approach [11], we used data from a large cohort under usual clinical follow-up; our results may thus indicate clinically relevant effects of CSII treatment on HbA1c under long-term and real-world conditions.

Historically, Norway has been an early adopter of CSII in the pediatric population; thus, it has a long experience with CSII use. The percentage of patients using CSII in Norway increased from $59 \%$ in 2010 , to $66 \%$ in 2013 , and $73 \%$ in 2017 [19]; meanwhile, the national mean HbA1c value declined from $8.3 \%$ $(67 \mathrm{mmol} / \mathrm{mol})$ in 2010 to $7.9 \%(63 \mathrm{mmol} / \mathrm{mol})$ in 2017. Looking at these data in isolation seems to imply that CSII improves glycemic control. However, looking at CSII use at a hospital level (ESM Fig. 1), no positive correlation between CSII use and HbA1c values are observed. Other unrecognized and unmeasured variables, such as increased diabetes education, improved follow-up structure in the clinic, and/ or the implementation of carbohydrate counting, could be confounders in this time period.
Given the limitations of a cross-sectional study design, our data do not support the hypothesis that insulin pumps available in 2017 achieved better metabolic control than MDI. It has been proposed that a low baseline HbA1c level leads to minimal changes, whereas a high baseline HbA1c level generally markedly lowers HbA1c levels in CSII users [28, 29]. Because our cohort had easy and early access to insulin pump treatment, right from diabetes onset in some cases, we were unable to validate this hypothesis. Access to technology might also explain why the results differ from those based on the SWEET registry data. In many countries, the access to technology is limited, which might lead to confounding [27]. Nevertheless, we expected a lower HbA1c in our pump user group, given the various possibilities of CSII compared with MDI. By contrast, only a small percentage of the cohort achieved the ISPAD's HbA1c goal of $<7.5 \%$, regardless of the use of an insulin pump.

\section{CSII Types}

In the LME model, we observed a $0.31 \mathrm{pp}$ $(3.2 \mathrm{mmol} / \mathrm{mol})$ lower HbA1c in users of older Medtronic Paradigm pumps and a $0.33 \mathrm{pp}$ $(3.6 \mathrm{mmol} / \mathrm{mol})$ lower HbA1c in the group of Omnipod users compared with the national recommended standard pump from Roche. These results are clinically surprising. Given the study design, these data have to be interpreted with caution. A possible explanation might be that users with an older pump reaching good results had no intention to change to a newer model. This might explain the lower HbA1c in the cohort with Medtronic Paradigm pumps. On the other hand, a highly motivated and experienced group of users might have wanted to try out new devices with a possible positive impact, and therefore adapted early to a patch pump such as the Omnipod. Regarding the newer Medtronic 640G pump, which can prevent low glucose values by automatically adjusting the basal insulin delivery [30], we expected a significantly lower reported incidence of severe hypoglycemia. However, this was not observed, likely due to the low number 
$(n=121)$ of users at the time. The user group that was given the Medtronic 640G pump at that time point might also have had a higher incidence of severe hypoglycemia episodes before. Notably, Omnipod users had a significantly lower incidence of diabetic ketoacidosis, which may be caused by the obligatory change of Omnipod infusion site every third day and its lack of possibility to be taken off intermittently.

\section{Continuous Glucose Monitoring}

The authors of the COMISAIR study, a 3-year, nonrandomized, prospective, real-world clinical trial following adults with type 1 diabetes, came to the conclusion that CGM was superior to SMBG in reducing HbA1c regardless of the insulin delivery method [31]. In a randomized clinical trial, Laffel et al. assessed the effect of CGM in adolescents and young adults and found an improvement in HbA1c of $0.4 \mathrm{pp}$ in CGM users compared to those using a blood glucose meter over a 26-week period [13]. These authors also noted that the proportion of CGM users in their CGM group who achieved ISPAD's HbA1c target of $<7.0 \%(53 \mathrm{mmol} / \mathrm{mol})$ or $7.5 \%$ $(58 \mathrm{mmol} / \mathrm{mol})$ did not increase. In the pediatric SWEET registry, a higher proportion of CGM users on MDI achieved an HbA1c of $<$ $7.0 \%(53 \mathrm{mmol} / \mathrm{mol})$, whereas pump and CGM users did not reach this goal more often than users of pumps without CGM [27]. Our findings provide clinically relevant information on the usage of CGM in children and adolescents with liberal access to CGM.

Because our CGM user group has a noteworthy younger age and shorter diabetes duration than the nonusers, adjusting the analyses for these confounders was critical. The adjusted LME model analyses revealed a small difference in HbA1c $(-0.18 \%$ or $-2 \mathrm{mmol} / \mathrm{mol})$ in the CGM group. Given the possibilities of alarms with high and low glucose values, we expected CGM users to have both lower HbA1c and a lower incidence of severe hypoglycemia and DKA. Thus, our data suggest that the incidence of severe hypoglycemia and DKA was independent of the general use of CGM.
CGM, nevertheless, has many advantages. The real-time monitoring enables the users or their family to intervene and act earlier than with intermittent measurements, and by this to regulate blood glucose better. The retrospective analysis of continuous glucose measurements supports healthcare professionals in finding patterns and thereby giving substantial advice to the user. However, CGM confronts both the user and the diabetes team with numerous data, which can be overwhelming, thereby necessitating new approaches to communicating diabetes care. Newer, more precise, accurate, and user-friendly systems, along with the combination of pumps with CGM in a closed-loop system, might help improve metabolic age in the near future.

\section{Strengths and Limitations}

Our study has a number of limitations. There is a potential selection bias in our cohort. On the one hand, the choice of CSII and CGM may be biased by local practice patterns and the personal preferences of the treating hospital and its healthcare professionals. On the other hand, the individual preference and the subjective needs of the patient and his/her family clearly influence the choice of treatment. The possibility of participating in the choice of insulin delivery system might be an essential part of empowerment, which is critical in diabetes care. Blair et al. [11] provided a good estimate of how these personal choices actually influence the resulting treatment. This "channeling bias" or "confounding by indication" is in no way documented or documentable and, therefore, is not possible to take into consideration. Furthermore, the cross-sectional design precludes the determination of the causative effects of a measure. However, if a large number of both CSII and CGM users had profited from these systems and had a higher baseline HbA1c, these users would be expected to be older and to have a longer diabetes duration. On the contrary, our CSII and CGM cohort comprised younger individuals with a shorter diabetes duration, and still, we see only a small difference in HbA1c values. Another potential limitation is that the 
HbA1c value clearly does not reflect every aspect of glycemic control. Knowing the percentage of glucose values in-range over a longer period in patients using CGM could contribute to the assessment of their metabolic control. However, these data would still not allow comparison with the results of non-CGM users.

Our study also has several strengths. It was a nationwide population-based study, with a high number of participants and a high level of completeness. Other strengths include the centrally analyzed HbA1c and the standardized registration and high usage of the different types of CSII and CGM devices. Our cohort with a long mean diabetes duration also had a high proportion of CSII and CGM use. We used an LME model with hospital as the random intercept. There is no possible treatment selection bias based on socioeconomic factors compared with situations in other countries [10, 26]. For the evaluation of rare acute complications, a large cross-sectional study is superior to studies with small sample sizes or short observational periods.

In conclusion, most children and adolescents with type 1 diabetes in our 2017 cohort did not achieve ISPAD's HbA1c goal, irrespective of whether they were using a CSII, a CGM, or neither. Both longitudinal and cross-sectional studies have limitations due to confounding factors, which makes the evaluation of the long-term effects of technical devices in diabetes treatment difficult.

\section{ACKNOWLEDGEMENTS}

We thank the laboratory staff at the Department of Medical Biochemistry Aker, Oslo University Hospital, Oslo, Norway, for the HbA1c analysis; the Norwegian Study Group for Childhood and Adolescent Diabetes representing the pediatric departments in Norway; and the patients and their parents for participating in NCDR.

Funding. This authors of this study have received funding from Helse Vest (\#912283, Heiko Bratke and \#911745, Pål R Njølstad), the
European Research Council (\#293574, Pål R Njølstad), the Norwegian Research Council (\#240413/F20, Pål R Njølstad), and the Novo Nordisk Fond (Pål R Njølstad). The Rapid Service Fee is covered by the Department of Research and Innovation, Haugesund Hospital, Fonna Health Trust, Haugesund, Norway.

Medical Writing and/or Editorial Assistance. The manuscript has been edited by Scribendi Editing and Proofreading Services.

Authorship. All named authors meet the International Committee of Medical Journal Editors (ICMJE) criteria for authorship for this article, take responsibility for the integrity of the work as a whole, and have given their approval for this version to be published.

Authorship Contributions. Heiko Bratke is the guarantor of this work. Heiko Bratke and Jörg Assmus had full access to all the data in the study and take responsibility for the integrity of the data and the accuracy of the data analysis. Heiko Bratke and Torild Skrivarhaug took the initiative for the study. Torild Skrivarhaug is responsible for data collection at the NCDR. Heiko Bratke, Jörg Assmus, and Torild Skrivarhaug participated in the study design and had access to the data.

Prior Presentation. Parts of this study were presented in poster form at the 45th Annual ISPAD Conference, Boston, 30 October-2 November 2019.

Disclosures. Heiko Bratke, Hanna D Margeirsdottir, Jörg Assmus, Pål R Njølstad, and Torild Skrivarhaug have nothing to disclose.

Compliance with Ethics Guidelines. To be registered in NCDR, all patients older than 11 years, and their parents have to sign an informed consent document. When the patients are 16 years, they have to re-sign the same informed consent document. Access to the data for this study was given to two of the authors (Heiko Bratke and Jörg Assmus), in accordance to the internal standards of the data owner (NCDR). The study was approved by the 
Regional Committee for Medical and Health Research Ethics (reference. no. 2016/1613/REC West, dated 11 November 2016), the data delivery committee of the Norwegian Childhood Diabetes Registry (NCDR, dated 8 May, 2017), and the Personal Data Security Office (Personvernombudet [PVO]) at Oslo University Hospital (dated 28 February 2017). The study was performed in accordance with the Helsinki Declaration of 1964 and its later amendments. The study was registered at clinicaltrials.gov (ref. no. NCT04201171).

Data Availability. The datasets generated during and/or analyzed during the current study are not publicly available due national data protection laws.

Open Access. This article is licensed under a Creative Commons Attribution-NonCommercial 4.0 International License, which permits any non-commercial use, sharing, adaptation, distribution and reproduction in any medium or format, as long as you give appropriate credit to the original author(s) and the source, provide a link to the Creative Commons licence, and indicate if changes were made. The images or other third party material in this article are included in the article's Creative Commons licence, unless indicated otherwise in a credit line to the material. If material is not included in the article's Creative Commons licence and your intended use is not permitted by statutory regulation or exceeds the permitted use, you will need to obtain permission directly from the copyright holder. To view a copy of this licence, visit http://creativecommons.org/licenses/by$\mathrm{nc} / 4.0 /$.

\section{REFERENCES}

1. DiMeglio LA, Acerini CL, Codner E, et al. ISPAD Clinical Practice Consensus Guidelines 2018: Glycemic control targets and glucose monitoring for children, adolescents, and young adults with diabetes. Pediatr Diabetes. 2018;19(S27):105-14.

2. Miller KM, Foster NC, Beck RW, et al. Current state of type 1 diabetes treatment in the U.S.: updated data from the T1D Exchange clinic registry. Diabetes Care. 2015;38(6):971-8.

3. Cengiz E, Xing D, Wong JC, et al. Severe hypoglycemia and diabetic ketoacidosis among youth with type 1 diabetes in the T1D Exchange clinic registry. Pediatr Diabetes. 2013;14(6):447-54.

4. Witsch M, Kosteria I, Kordonouri O, et al. Possibilities and challenges of a large international benchmarking in pediatric diabetology-the SWEET experience. Pediatr Diabetes. 2016;17(Suppl 23): 7-15.

5. Charalampopoulos D, Hermann JM, Svensson J, et al. Exploring variation in glycemic control across and within eight high-income countries: a crosssectional analysis of 64,666 children and adolescents with type 1 diabetes. Diabetes Care. 2018;41(6):1180-7.

6. McMahon SK, Airey FL, Marangou DA, et al. Insulin pump therapy in children and adolescents: improvements in key parameters of diabetes management including quality of life. Diabet Med. 2005;22(1):92-6.

7. Joshi KK, Haynes A, Smith G, Jones TW, Davis EA. Comparable glycemic outcomes for pediatric type 1 diabetes patients in metropolitan and nonmetropolitan regions of Western Australia: A population-based study. Pediatr Diabetes. 2018;19(3): 486-92.

8. Skrivarhaug T, Kummernes SJ, Kamaleri Y. The Norwegian Childhood Diabetes Registry (NCDR) annual report 2018. Available at: https://oslouniversitetssykehus.no/seksjon-avdeling/ Documents/2019.10.01_Endelig_Årsrapport_2018. pdf $\#$ page $=1$. Accessed 5 Aug 2021 .

9. Szypowska A, Schwandt A, Svensson J, et al. Insulin pump therapy in children with type 1 diabetes: analysis of data from the SWEET registry. Pediatr Diabetes. 2016;17(Suppl 23):38-45.

10. Sherr JL, Hermann JM, Campbell F, et al. Use of insulin pump therapy in children and adolescents with type 1 diabetes and its impact on metabolic control: comparison of results from three large, transatlantic paediatric registries. Diabetologia. 2016;59(1):87-91.

11. Blair JC, McKay A, Ridyard C, et al. Continuous subcutaneous insulin infusion versus multiple daily injection regimens in children and young people at diagnosis of type 1 diabetes: pragmatic randomised controlled trial and economic evaluation. BMJ. 2019;365:L1226.

12. Bergenstal RM, Tamborlane WV, Ahmann A, et al. Effectiveness of sensor-augmented insulin-pump 
therapy in type 1 diabetes. $\mathrm{N}$ Engl J Med. 2010;363(4):311-20.

13. Laffel LM, Kanapka LG, Beck RW, et al. Effect of continuous glucose monitoring on glycemic control in adolescents and young adults with type 1 diabetes: a randomized clinical trial. JAMA. 2020;323(23):2388-96.

14. Charleer S, Mathieu C, Nobels F, et al. Effect of continuous glucose monitoring on glycemic control, acute admissions, and quality of life: a realworld study. J Clin Endocrinol Metab. 2018;103(3): 1224-32.

15. Aberer F, Hajnsek M, Rumpler M, et al. Evaluation of subcutaneous glucose monitoring systems under routine environmental conditions in patients with type 1 diabetes. Diabetes Obes Metab. 2017;19(7): 1051-5.

16. Buckingham BA, Bailey TS, Christiansen M, et al. Evaluation of a predictive low-glucose management system in-clinic. Diabetes Technol Ther. 2017;19(5):288-92.

17. Riemsma R, Corro Ramos I, Birnie R, et al. Integrated sensor-augmented pump therapy systems [the MiniMed(R) Paradigm Veo system and the Vibe and G4(R) PLATINUM CGM (continuous glucose monitoring) system] for managing blood glucose levels in type 1 diabetes: a systematic review and economic evaluation. Health Technol Assess. 2016;20(17):v-Xxxi, 1-251.

18. Vesco AT, Jedraszko AM, Garza KP, WeissbergBenchell J. Continuous glucose monitoring associated with less diabetes-specific emotional distress and lower A1c among adolescents with type 1 diabetes. J Diabetes Sci Technol. 2018;12(4):792-9.

19. Skrivarhaug T, Kummernes SJ, Drivvoll AK. The Norwegian Childhood Diabetes Registry (NCDR) annual report 2017. Available at: https://oslouniversitetssykehus.no/avdelinger/Documents/ 2018.10.20_Endelig\%20Årsrapport\%202017.pdf. Accessed 5 Aug 2021.

20. Rewers MJ, Pillay K, de Beaufort C, et al. ISPAD Clinical Practice Consensus Guidelines 2014. Assessment and monitoring of glycemic control in children and adolescents with diabetes. Pediatr Diabetes. 2014;15(Suppl 20):102-14.

21. Ly TT, Maahs DM, Rewers A, Dunger D, Oduwole A, Jones TW. ISPAD Clinical Practice Consensus Guidelines 2014. Assessment and management of hypoglycemia in children and adolescents with diabetes. Pediatr Diabetes. 2014;15(Suppl 20): 180-92.

22. Wolfsdorf JI, Allgrove J, Craig ME, et al. ISPAD Clinical Practice Consensus Guidelines 2014. Diabetic ketoacidosis and hyperglycemic hyperosmolar state. Pediatr Diabetes. 2014;15(Suppl 20):154-79.

23. R Core Team. R: a language and environment for statistical computing. Vienna: R Foundation for Statistical Computing; 2019. Available at: http:// www.r-project.org/index.html. Accessed 5 Aug 2021.

24. Pinheiro J BD, DebRoy S, Sarkar D, R Core Team. nlme: linear and nonlinear mixed effects models. $\mathrm{R}$ package version 3.1-148. 2020. https://CRAN.Rproject.org/package=nlme.

25. Lenth R. Emmeans: estimated marginal means, aka least-squares means. R package version 1.4.7. 2020. Available at: https://CRAN.R-project.org/package= emmeans. Accessed 5 Aug 2021.

26. Burckhardt MA, Smith GJ, Cooper MN, Jones TW, Davis EA. Real-world outcomes of insulin pump compared to injection therapy in a populationbased sample of children with type 1 diabetes. Pediatr Diabetes. 2018;19(8):1459-66.

27. Cardona-Hernandez R, Schwandt A, Alkandari H, et al. Glycemic outcome associated with insulin pump and glucose sensor use in children and adolescents with type 1 diabetes. Data from the international pediatric registry SWEET. Diabetes Care. 2021;44(5):1176-84.

28. Pickup JC. Is insulin pump therapy effective in Type 1 diabetes? Diabet Med. 2019;36(3):269-78.

29. Pickup JC, Sutton AJ. Severe hypoglycaemia and glycaemic control in Type1 diabetes: meta-analysis of multiple daily insulin injections compared with continuous subcutaneous insulin infusion. Diabet Med. 2008;25(7):765-74.

30. Abraham MB, Jones TW, Naranjo D, et al. ISPAD Clinical Practice Consensus Guidelines 2018: assessment and management of hypoglycemia in children and adolescents with diabetes. Pediatr Diabetes. 2018;19(Suppl 27):178-92.

31. Šoupal J, Petruželková L, Grunberger G, et al. Glycemic outcomes in adults with T1D Are impacted more by continuous glucose monitoring than by insulin delivery method: 3 years of follow-up from the COMISAIR study. Diabetes Care. 2020;43(1): 37-43. 